\title{
In Other Words
}

\author{
Pierre Bourdieu
}


In Memory of my Father 


\section{In Other Words}

Essays Towards a Reflexive Sociology

\section{Pierre Bourdieu}

Translated by Matthew Adamson

Stanford University Press

Stanford, California 
Stanford University Press

Stanford, California

(C) Chapters 1, 2, 3, 4, 5, 6, 8, 9, 10, 11, 12, Les Editions de Minuit 1987; Chapter 7, Pierre Bourdieu; Chapter 13, Les Editions de Minuit 1982; Bibliography, Yvette Delsaut

English translation (except Chapter 7 and Bibliography) (C) 1990 Polity Press, Cambridge; English translation of Chapter 7 (C) 1990 Loic J. D. Wacquant with Matthew Lawson

Originating publisher of English edition:

Polity Press, Cambridge, in association with Basil Blackwell, Oxford

First published in the U.S.A. by Stanford University Press, 1990

Cloth ISBN 0-8047-1557-2

Paper ISBN 0-8047-1725-7

LC 88-63436 